\title{
Methodology for the analysis of voltage unbalance in networks with single-phase distributed generation
}

DOI:

10.1049/iet-gtd.2016.1155

\section{Document Version}

Accepted author manuscript

Link to publication record in Manchester Research Explorer

\section{Citation for published version (APA):}

Liao, H., \& Milanovi, J. V. (2017). Methodology for the analysis of voltage unbalance in networks with single-phase distributed generation. IET Generation, Transmission and Distribution, 11(2), 550-559. https://doi.org/10.1049/ietgtd.2016.1155

\section{Published in:}

IET Generation, Transmission and Distribution

\section{Citing this paper}

Please note that where the full-text provided on Manchester Research Explorer is the Author Accepted Manuscript or Proof version this may differ from the final Published version. If citing, it is advised that you check and use the publisher's definitive version.

\section{General rights}

Copyright and moral rights for the publications made accessible in the Research Explorer are retained by the authors and/or other copyright owners and it is a condition of accessing publications that users recognise and abide by the legal requirements associated with these rights.

\section{Takedown policy}

If you believe that this document breaches copyright please refer to the University of Manchester's Takedown Procedures [http://man.ac.uk/04Y6Bo] or contact uml.scholarlycommunications@manchester.ac.uk providing relevant details, so we can investigate your claim.

\section{OPEN ACCESS}




\title{
Methodology for the Analysis of Voltage Unbalance in Networks with Single Phase Distributed Generation
}

\author{
Huilian Liao ${ }^{1}$, Jovica V. Milanović ${ }^{1^{*}}$ \\ ${ }^{1}$ School of Electrical and Electronic Engineering, The University of Manchester, Manchester, \\ M60 1QD, UK \\ milanovic@manchester.ac.uk
}

\begin{abstract}
This paper presents a sequence networks based methodology for investigating voltage unbalance in distribution networks with renewable generation. The sequence networks are derived from the original asymmetrical three-phase network, and then interconnected to study sequence voltages and unbalance propagation through the network. The approach enables to analyse the influence of line impedance, load demand and network topology on voltage unbalance caused by distributed generation in the network. The critical factors which impact the unbalance severity and the propagation mode are also identified. The approach is validated by comparing calculated sequence voltages with the results obtained by phase voltage based methodology.
\end{abstract}

\section{Introduction}

Voltage unbalance, as a common phenomenon found in a three-phase power system, causes additional power losses and damage to power system equipment, rotating plant and user connected devices, resulting in substantial financial losses to both Distribution Network Operators (DNOs) and end customers. This issue is one of the critical power quality problems, and it is becoming a major focal point for utilities and distribution generation industries [1]. The asymmetry in the network typically appears as a result of the connection of single-phase customer, which creates uneven load among phases. The asymmetry gets further aggravated when single-phase distribution generation (DG) is integrated into the existing distribution networks [2]. With the increasing penetration of non-dispatchable renewable energy in power systems, especially in microgrids where renewable energy sources gradually eclipse today's more common conventional sources of diesel and natural gas, the quality of supply, including voltage unbalance, is still one of the greatest technical and operation challenges [3]. Voltage unbalance issues have been addressed mainly by using automated controls and other appropriate technologies in the past [4]. However, these issues can also be mitigated by comprehensive analysis and careful planning. The understanding of unbalance propagation and identification of critical factors that affect the unbalance phenomena caused by the connection of DGs are still needed.

Voltage unbalance factor (VUF), defined as the ratio of negative to positive sequence voltage, is commonly used to assess the unbalance severity at buses [5,6]. The two symmetrical components (i.e., negative and positive sequence voltages) can be obtained using phase voltage based methodology, in which phase voltage of each bus is obtained using Newton-Raphson or Gauss-Seidel methods [7] or basic 
Ohm's law [8]. The derived phase voltages are then post-processed by decomposing them into symmetrical components, and used for final unbalance factor calculation. Most commercially available software including DIgSILENT/PowerFactory employs phase voltage based methodology for unbalance calculation under normal operating condition. Alternatively, the symmetrical components can also be obtained using Fortescue transform method, in which three-phase components are decomposed into a new set of symmetrical components [9] which are then used to form the equivalent balanced three-phase circuits, i.e., sequence networks. The advantage of this transformation is that the unbalanced power system is separated into three uncoupled networks. It simplifies the analysis of unbalance and avoids checking numerous load flow results. Fortescue transform method plays an essential role in analysing power system faults and explaining some power system phenomena. For this purpose, models of various components have been developed to construct the sequence networks [10,11].

Most past unbalance studies in literature are concerned with unbalance loads and asymmetric lines [7, $8,12-14]$. In [13], sequence networks are used to analyse the propagation of unbalance phenomena caused by unbalance loads on a simple three-bus electric power system. With the growing integration of distributed generation and increasing severity of unbalance phenomenon in existing distribution networks, it is necessary to study the impact of DG on unbalance severity and unbalance propagation, and to understand the difference between the unbalance phenomena caused by DG and those caused by other asymmetrical components in the network. Especially in DG integration planning, understanding the propagation mode of unbalance phenomena is essential. In [15], a network with two shunt-connected impedances is used to represent the negative-sequence network of a power system with connected DG. In literature, sequence network models of DG were developed [10], however they are mainly used for shortcircuit analysis rather than unbalance propagation analysis. Comprehensive study on the propagation mode of unbalance phenomena caused by DG and the potential factors that will impact the unbalance propagation is still limited in literature, in particular the use of sequence network analysis for this purpose.

This paper presents a sequence network analysis based methodology, in which separate single-line diagrams are developed for each of the positive-, negative- and zero-sequence systems using the method of symmetrical components. With this methodology, the propagation of symmetrical components of unbalanced voltage can be straightforwardly presented using sequence networks. This methodology avoids numerous load flow calculations and inherits its simplicity from Fortescue transform method. By analysing the derived sequence circuit equations the paper studies the impact of network parameters/settings (including line impedance, load demand and network topology) on the unbalance severity and reveals the relationship between the sequence components and the open circuit voltage from 
the perspective of its influence on unbalance. Finally, the paper proposes a sequence network based formulae to identify the critical factors that affect the propagation of unbalance caused by DG, and comprehensively analyses the impact of various network components on the unbalance severity and its propagation. This analysis approach can assist network planners and operators in understanding the unbalance propagation in the networks, identifying the critical factors that should be taken into account and accordingly choosing the appropriate location for renewable energy integration in order to minimise the effect of unbalance propagation.

\section{Sequence Networks Based Methodology}

The connection of single-phased DG is illustrated in Fig. 1(a). Due to voltage related issues small DG is usually required to operate with a fixed power factor or to have fixed reactive power control [16]. Since the connection of a single-phase generator results in the injection of unbalanced current [15], DG can be considered as an unbalance current source at the connecting point.

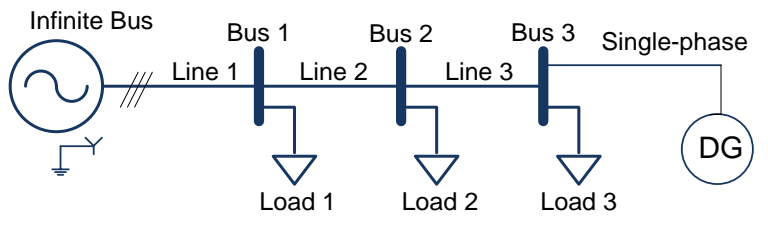

$a$
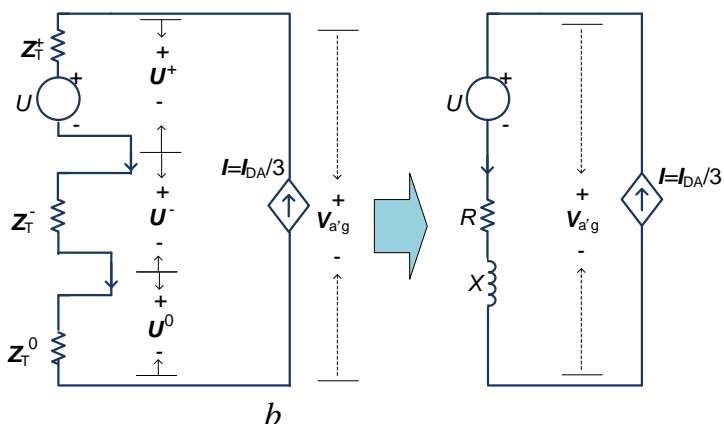

Fig. 1. Three-phase feeder with a single-phase connected DG a Single-line diagram

b Thévenin equivalent circuits

Assume the single-phased DG is connected between point $a^{\prime}$ at phase A and reference point $g$. The current injected by DG (i.e. the injected current at phase A) can be obtained by:

$$
\boldsymbol{I}_{\mathrm{DA}}=\left(\boldsymbol{S}_{\mathrm{DG}} / \boldsymbol{V}_{a^{\prime} g}\right)^{*}
$$

The currents in phases B and C are denoted as $\boldsymbol{I}_{\mathrm{DB}}$ and $\boldsymbol{I}_{\mathrm{DC}}$ respectively, where $\boldsymbol{I}_{\mathrm{DB}}=\boldsymbol{I}_{\mathrm{DC}}=0$. This unbalanced current source injects the same amount of positive-, negative-, and zero-sequence currents to the network. The symmetrical components of this current source can be obtained by:

$$
I_{\mathrm{DA}}^{+}=I_{\mathrm{DA}}^{-}=I_{\mathrm{DA}}^{0}=\frac{I_{\mathrm{DA}}}{3}
$$

Steady-state sequence models of various components (including generators and loads) have been developed for fault analysis in literature. Based on these developed models, items that have different impedances for different phase sequences are identified and used to construct sequence networks 
respectively. The unbalance of the distribution lines is modelled and addressed using positive-, negativeand zero-sequence impedances [17], i.e., $Z^{+,} Z$ and $Z^{0}$ in Fig. 1(b). The voltage between points $a^{\prime}$ and $g$, denoted by $\boldsymbol{V}_{a^{\prime} g}$, can be described as the combination of its three symmetrical components as below:

$$
\boldsymbol{V}_{a^{\prime} g}=\boldsymbol{V}_{a^{\prime} g}^{+}+\boldsymbol{V}_{a^{\prime} g}^{-}+V_{a^{\prime} g}^{0}
$$

where $\boldsymbol{V}_{a^{\prime} g}^{+}, \boldsymbol{V}_{a^{\prime} g}^{-}$and $\boldsymbol{V}_{a^{\prime} g}^{0}$ represent the positive-, negative- and zero-sequence voltages respectively. Based on (2) and (3), it can be derived that $\boldsymbol{V}_{a^{\prime} g}$ is the voltage across the series connection of the positive-, negative- and zero-sequence networks while current $I_{\mathrm{DA}} / 3$ flows through this series connection. Based on this, the equivalent circuit of Fig. 1(a) can be represented by its sequence networks where a voltage controlled current source is connected in series with the three sequence networks. Replacing the three sequence networks with their Thévenin equivalent circuits, a simplified circuit can be obtained, as shown in Fig. 1(b). This circuit includes only three components, i.e., the open circuit voltage between points $a^{\prime}$ and $g$ in the positive-sequence network, denoted by $U$, the total equivalent impedance of the series connection of the three sequence networks, denoted by $\boldsymbol{Z}_{\mathrm{T}}=R+j X$, and the voltage controlled current source, which generates current $\frac{I_{\mathrm{DA}}}{3}$.

For the convenience of analysis, a simplifying procedure is introduced here: DG is connected to the network with a zero reactive power, i.e. $\boldsymbol{S}_{\mathrm{DG}}=P$; the reference angle of the voltages is based on $\boldsymbol{U}$, whose angle is $0^{\circ}$, i.e. $\boldsymbol{U}=U$; the reference magnitude of the voltages is based on $\boldsymbol{E}$, whose voltage magnitude is 1p.u. It can be derived from Fig. 1(b) that:

$$
\boldsymbol{U}+\boldsymbol{Z}_{\mathrm{T}} \boldsymbol{I}=\boldsymbol{V}_{a^{\prime} g}
$$

$\boldsymbol{V}_{a^{\prime} g}$ can be represented using complex format as below:

$$
\boldsymbol{V}_{a^{\prime} g}=U_{1}+j U_{2}
$$

Based on (1), (5) and the aforementioned assumption, $I$ can be represented as a function of $P, U_{1}$ and $U_{2}$ :

$$
\boldsymbol{I}=\frac{1}{3}\left(\frac{P}{V_{a^{\prime} g}}\right)^{*}=\frac{P}{3\left(U_{1}-j U_{2}\right)}
$$

Replacing $\boldsymbol{U}, \boldsymbol{Z}_{\mathrm{T}}, \boldsymbol{I}$ and $\boldsymbol{V}_{a^{\prime} g}$ of (4) with $U, R+j X,(6)$ and (5) respectively, (4) can be rewritten as:

$$
U+\frac{P(R+j X)}{3\left(U_{1}-j U_{2}\right)}=U_{1}+j U_{2}
$$

The imaginary part of (7) yields $3 U U_{2}=P X$, which can be reformatted as:

The real part of (7) yields:

$$
U_{2}=\frac{P X}{3 U}
$$

$$
3 U_{1}^{2}-3 U U_{1}-P R+3\left(\frac{P X}{3 U}\right)^{2}=0
$$


Solving (9), $U_{1}$ can be presented as below:

$$
U_{1}=\frac{3 U \pm \sqrt{9 U^{2}+12 P R-36\left(\frac{P X}{3 U}\right)^{2}}}{6}
$$

The selection of the sign in (10) is based on the following analysis. In the sequence networks, load impedances and generator impedances are connected in shunt, and generators impedances are much smaller than load impedances. Therefore, loads do not appreciably affect the total impedance $\boldsymbol{Z}_{\mathrm{T}}$. Since the generator impedances and line impedances are usually small, $\boldsymbol{Z}_{\mathrm{T}}$ is small consequently. With the relatively small $\boldsymbol{Z}_{\mathrm{T}}, \boldsymbol{V}_{a^{\prime} g}$ is mainly determined by $U$. In distribution networks where cables are deployed, usually the line resistance is larger than line reactance. In other words, the influence of line resistance is larger compared to line reactance, and $\boldsymbol{V}_{a^{\prime} g}$ is mainly determined by the real part of $\boldsymbol{V}_{a^{\prime} g}$, i.e. $U_{1}$. Based on the analysis above, it can be concluded that the magnitude of $U_{1}$ and $U$ should be close, thus sign ' + ' is selected in (10). Then, the magnitude of voltage $\boldsymbol{V}_{a^{\prime} g}$ can be derived based on (8) and (10) as below:

$$
\left|\boldsymbol{V}_{a^{\prime} g}\right|=\sqrt{U_{1}^{2}+U_{2}^{2}}=\sqrt{\frac{U^{2}}{2}+\frac{P R}{3}+\frac{U}{6} \sqrt{9 U^{2}+12 P R-4\left(\frac{P X}{U}\right)^{2}}}
$$

Since voltage unbalance is mainly concerned with symmetrical components of voltages (in particular positive- and negative-sequence voltages), the three symmetrical components of $\boldsymbol{V}_{a^{\prime} g}$ can be obtained by applying (6) on Fig. 1(b):

$$
\begin{aligned}
\boldsymbol{U}^{+} & =U+\frac{P}{3\left(U_{1}-j U_{2}\right)} \boldsymbol{Z}^{+} \\
\boldsymbol{U}^{-} & =\frac{P}{3\left(U_{1}-j U_{2}\right)} \boldsymbol{Z}^{-} \\
\boldsymbol{U}^{0} & =\frac{P}{3\left(U_{1}-j U_{2}\right)} \boldsymbol{Z}^{0}
\end{aligned}
$$

\subsection{Correlation between variation in symmetrical components and open circuit voltage $U$}

Depending on the DG outputs, the variation of network parameters results in a change of the symmetrical components of the unbalance voltage. This influence varies depending on the type of the network parameter. This section studies the variation of symmetrical components by considering the open circuit voltage $U$, and identifies the network parameters which have the highest impact on the voltage unbalance. 
2.1.1 The effect of variation of line impedance: Given fixed DG output, the variation of network parameters results in the variation of the magnitude of symmetrical components of $\boldsymbol{V}_{a^{\prime}} g$. The impact of the variation of line impedance on $\left|\boldsymbol{U}^{-}\right|,\left|\boldsymbol{U}^{+}\right|$and $\left|\boldsymbol{U}^{0}\right|$ is analysed here based on (11)-(14). Since network parameters are modified, open circuit voltage $U$ varies accordingly. If line impedance $Z_{\text {Linei }}$ increases, $U$ in positive-sequence network will decrease accordingly.

Based on (11) and $\left|\boldsymbol{U}^{-}\right|=\frac{P}{\left|\boldsymbol{V}_{a^{\prime} g}\right|} \frac{\left|\boldsymbol{Z}^{-}\right|}{3}$ which can be derived from (13), $\left|\boldsymbol{U}^{-}\right|$can be rewritten as:

$$
\left|\boldsymbol{U}^{-}\right|=\frac{P}{\sqrt{\frac{U^{2}}{2}+\frac{P R}{3}+\frac{U}{6} \sqrt{9 U^{2}+12 P R-4\left(\frac{P X}{U}\right)^{2}}}} \frac{\left|\boldsymbol{Z}^{-}\right|}{3}
$$

Assume the resistance component in $\boldsymbol{Z}_{\mathrm{Line} i}$ varies. Once $\boldsymbol{Z}_{\mathrm{Line} i}$ is changed, both impedance and $U$ in (15) vary accordingly. Based on the potential division calculation performed on positive-sequence network and the voltage magnitude derived from (15), it can be derived that the variation of $U$, as a response to the variation of line impedance, has larger influence on $\left|\boldsymbol{U}^{-}\right|$, compared to the variation of $R, X$ and $\left|Z^{-}\right|$in (15), assuming that the variables fall into reasonable ranges, e.g., $U \in[3,13] \mathrm{kV}, P \in[0.1,5] \mathrm{MW}, R \in$ $[0.2,10] \mathrm{Ohm}$, and $X \in[0.1,5] \mathrm{Ohm}$, which are the possible ranges considered in the study [18]. This will be further demonstrated in Section 3.1. Based on the analysis above, it can be seen that as $Z_{\mathrm{Line} i}$ increases, $U$ decreases, and consequently $\left|\boldsymbol{U}^{-}\right|$increases. As for $\left|\boldsymbol{U}^{+}\right|$, the first term of (12), i.e. $U$, is the main factor which determines the variation of $\left|\boldsymbol{U}^{+}\right| .\left|\boldsymbol{U}^{+}\right|$decreases as $U$ decreases. In other words, $\left|\boldsymbol{U}^{+}\right|$decreases as line impedance increases.

2.1.2 The effect of variation of load demand: If loads (three phase balanced load) are modelled as constant power loads, the larger constant power load will draw larger current from the network (due to the relatively steady voltage), i.e., the corresponding load impedance is smaller. Similarly, if loads are modelled as constant impedance, the load with larger rated demand has smaller impedance. Therefore, the increase in load demand results in the decrease in the equivalent impedance of the corresponding load. Applying potential division to positive-sequence network, it can be derived that $U$ decreases as $\boldsymbol{Z}_{\text {Load } i}$ decreases. The procedure used to analyse line impedance variation applies here, and the same conclusion can be obtained, i.e. although the variation of load impedance results in the variation of $U$ and impedance in (15), the variation of $U$ has larger influence on $\left|\boldsymbol{U}^{-}\right|$compared to the variation of impedance in (15). $\left|\boldsymbol{U}^{-}\right|$and $\left|\boldsymbol{U}^{0}\right|$ increase as $U$ decreases, the variation trend of $\left|\boldsymbol{U}^{+}\right|$is the same as that of $U$. In other words, as load demand increases, both $\left|\boldsymbol{U}^{-}\right|$and $\left|\boldsymbol{U}^{0}\right|$ increase, while $\left|\boldsymbol{U}^{+}\right|$decreases. Although the sequence 
voltages in response to the variation of line impedance and in response to the load demand change is similar, line impedance has larger influence on sequence voltages compared with load variation, due to the fact that line impedance is much smaller than load impedance, and the open circuit voltage $U$ and the Thévenin equivalent impedances, i.e. $\left|Z^{+}\right|$and $\left|Z^{-}\right|$, are mainly determined by line impedances rather than load impedances. In other words, the variation of symmetrical component $\boldsymbol{Z}_{\text {Linei }}$ contributes more to voltage unbalance than the variation of symmetrical component $\boldsymbol{Z}_{\text {Load } i}$.

2.1.3 The effect of variation of network topology: If another branch is added to the original network, as shown in Fig. 2(a), its Thévenin equivalent impedance, denoted by $Z_{\mathrm{BTh}}$, can be obtained and connected to the original sequence networks in shunt with other loads, as shown in Fig. 2(b). Adding a new branch to the original network is similar to adding a new load at the connecting point. The analysis of the topology change is the same as that of load demand variation as discussed above. It can be derived that if a new branch is added to the original network, $U$ will decrease, consequently $\left|\boldsymbol{U}^{-}\right|$and $\left|\boldsymbol{U}^{0}\right|$ will increase accordingly, and $\left|\boldsymbol{U}^{+}\right|$will decrease. Similar to the influence of load demand variation, the influence of the new network topology on sequence voltages is much smaller than that of line impedances.

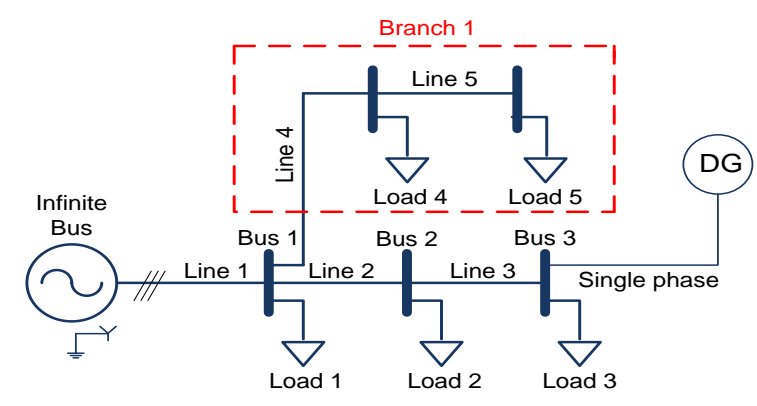

$a$

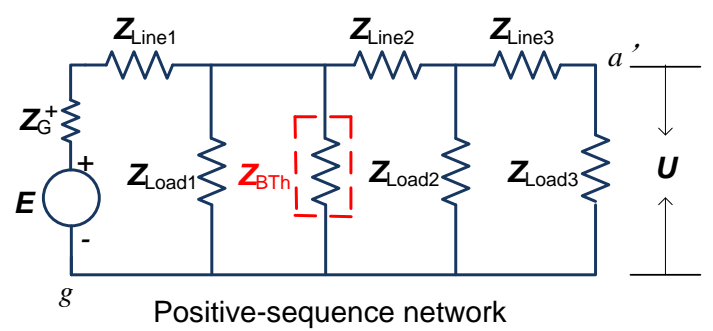

$b$

Fig. 2. Connection of a new feeder and its positive-sequence network a Original network connected with a new feeder b Positive-sequence network

The presented analysis can facilitate better DG planning in terms of the selection of DG connection points. Prior to the connection of a single-phase DG, assuming balanced network conditions, only the positive-sequence network is affected, as $I$ in (4) is relatively small, and the influence of negative- and zero-sequence networks is negligible. The open circuit voltage of the positive-sequence network $(U)$ can be determined by measuring open circuit voltage at the potential DG connection point and the trend of variation of the symmetrical components, as a result of the connection of a DG, can be estimated following the presented correlation analysis. 


\subsection{Analysis of propagation of negative-sequence voltage using sequence networks}

The negative-sequence network is given in Fig. 3(a), together with the phasor diagram of the current flowing through line impedances. Since $\boldsymbol{Z}_{\text {Load }}$ is much larger than $\boldsymbol{Z}_{\text {Line }}$, majority of negative-sequence current flows through line impedances, while small portion is divided into the path connected with loads. The currents flowing through $\boldsymbol{Z}_{\mathrm{Line} 1}, \boldsymbol{Z}_{\mathrm{Line} 2}$ and $\boldsymbol{Z}_{\mathrm{Line} 3}$ are denoted as $\boldsymbol{I}_{1}, \boldsymbol{I}_{2}$ and $\boldsymbol{I}_{3}$ respectively. It can be seen from the phasor diagram that the angle of the current on the left of the sequence network is slightly smaller than that on the right of the network. The negative-sequence current propagates from the DG connection point to the infinite bus (equivalent generator). $\left|\boldsymbol{U}^{-}\right|$reaches its maximum value at the DG connected point, and gradually decreases along the path to the equivalent generator. If the three line impedances vary with the same amount, their influence on the variation of $\left|\boldsymbol{U}^{-}\right|$follows the following order: $\boldsymbol{Z}_{\text {Line3 }}, \boldsymbol{Z}_{\mathrm{Line} 2}$ and $\boldsymbol{Z}_{\mathrm{Line} 1}$, due to $\left|\boldsymbol{I}_{3}\right|>\left|\boldsymbol{I}_{2}\right|>\left|\boldsymbol{I}_{1}\right|$. If $\boldsymbol{Z}_{\text {Line3 }}$ is much larger than $\boldsymbol{Z}_{\mathrm{Line} 2}$ and $\boldsymbol{Z}_{\mathrm{Line} 1}$, the negative-sequence voltage will be greatly reduced when it propagates from $\left|\boldsymbol{V}_{3}\right|$ to $\left|\boldsymbol{V}_{2}\right|$. It can be seen that the propagation is greatly affected by the distribution of the line impedances along the feeder.

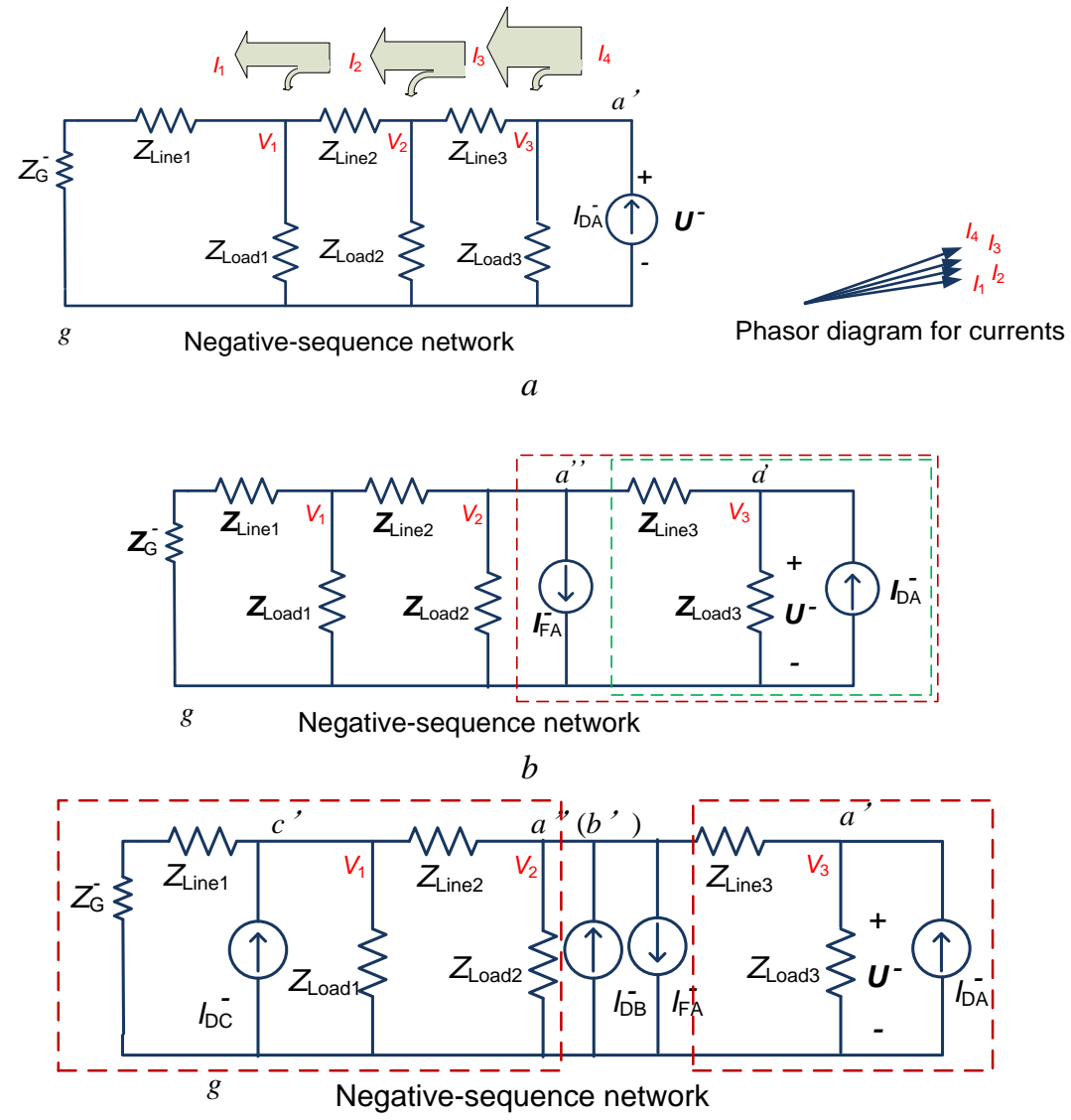

(c)

Fig. 3. Illustration of the propagation of negative-sequence voltage 
a Propagation $\boldsymbol{U}^{-}$and phasor diagram of currents

b The negative-sequence network with DG and unbalance load

C The negative-sequence network with three DGs and unbalance load

Consider an unbalanced load (Load 3) in the network, whose load demand at phase A is different from that at phases B and C, is connected at point $a^{\prime \prime}$. Assume the load impedance of Load 3 at phase A is $\boldsymbol{Z}_{\mathrm{Load} 3}^{\prime \prime}$, and that of both phases B and C is $\boldsymbol{Z}_{\mathrm{Load} 3}$. Based on sequence network analysis, load impedance $\boldsymbol{Z}_{\mathrm{Load3}}^{\prime \prime}$ is replaced with the shunt connection of $\boldsymbol{Z}_{\mathrm{Load} 3}$ and $\boldsymbol{Z}_{\mathrm{F}}$. The same procedure applied for analysing DG connection applies here as well. If the extra load demand at phase A is modelled as the injection of a constant power, the current flowing through the negative-sequence impedance, denoted by $\boldsymbol{I}_{\mathrm{FA}}^{-}$, can be obtained from:

$$
\boldsymbol{I}_{\mathrm{FA}}^{-}=\boldsymbol{I}_{\mathrm{F}} / 3=\frac{1}{3}\left(\boldsymbol{S}_{\mathrm{F}} / \boldsymbol{V}_{a^{\prime \prime} g}\right)^{*}
$$

The three sequence networks can be interconnected using:

$$
\boldsymbol{V}_{a^{\prime \prime} g}=\boldsymbol{V}_{a^{\prime \prime} g}^{+}+\boldsymbol{V}_{a^{\prime \prime} g}^{-}+\boldsymbol{V}_{a^{\prime \prime} g}^{0}
$$

Assume now that a DG is connected at point $a^{\prime}$ while the unbalance load is connected at another point, $a^{\prime \prime}$. The negative-sequence network can be obtained as given in Fig. 3(b). The relationship between the voltage at the connected point $\boldsymbol{a}^{\prime}$ and $\boldsymbol{I}_{\mathrm{DA}}^{-}$can be obtained using (2), while the relationship between the voltage at the connected point $a^{\prime \prime}$ and $\boldsymbol{I}_{\mathrm{FA}}^{-}$can be obtained using (16). The relationship among the three sequence networks is represented by both (3) and (17). With these formulae, the sequence voltages throughout the network can be calculated.

With the available $I_{\mathrm{DA}}^{-}$and $\boldsymbol{I}_{\mathrm{FA}}^{-}$, negative-sequence network can be used to study the propagation of negative-sequence voltage directly. It can be seen from Fig. 3(b) that the negative-sequence voltage propagates from $a^{\prime \prime}$ to the left part of the circuit, and its decrease rate is mainly dependent on the line impedances along the propagation path. To see the unbalance severity at point $a$ ", the circuit within the dashed green box is replaced by its Norton equivalent circuit, i.e. the shunt connection between a current source denoted as $\boldsymbol{I}_{\mathrm{DA}}^{-\prime}$ and an impedance. The difference between $\boldsymbol{I}_{\mathrm{DA}}^{-\prime}$ and $\boldsymbol{I}_{\mathrm{FA}}^{-}$determines the unbalance severity at points $a^{\prime \prime}$, together with the total equivalent impedance of the negative-sequence circuit seen from the two ports $a^{\prime \prime}$ and $g$. The same analysis procedure can be followed to analyse the unbalance severity at point $a^{\prime}$.

Furthermore, consider a more generic scheme where multiple DGs are connected to different phases at different nodes. Based on the case in Fig. 3(b), another two single-phase DGs are connected at points $b^{\prime}$ (at phase B) and $c^{\prime}$ (at phase $\mathrm{C}$ ) respectively. The negative-sequence network constructed for this network 
is given in Fig. 3(c), which shows that the three DGs are connected at different nodes in the network. Variables $\boldsymbol{I}_{\mathrm{DB}}^{-}$and $\boldsymbol{I}_{\mathrm{DC}}^{-}$can be related to the voltages at the connected point $b^{\prime}$ and $c^{\prime}$ respectively using (2). The same analysis approach, i.e., the analysis conducted for Fig. 3(b), is applied to analyse the propagation of negative-sequence voltage throughout the network. To see the unbalance severity at point $a^{\prime \prime}$, the circuits within the red dashed lines can be replaced by their Norton equivalent circuits. The combination of $I_{\mathrm{FA}}^{-}, \boldsymbol{I}_{\mathrm{DB}}^{-}$and the equivalent current sources within the dashed lines determines the unbalance severity at point $a^{\prime \prime}$, together with the equivalent impedances. The same analysis procedure can be adopted to analyse the unbalance severity at other nodes.

\section{Simulation Studies}

Two feeders with a single-phase DG connected at bus B7, as shown in Fig. 4(a), are adopted for study here. The topologies of the two feeders are similar, except that there are two extra branches in Fig. 4(b), marked by red dashed lines. It can be seen that in total three extra buses and three more lines are added in Fig. 4(b). The line impedances are modeled using the actual parameters of underground cables. The two feeders are simulated in DIgSILENT/PowerFactory, in which the sequence voltages are obtained by post-processing the phase voltages that are derived from load flow calculation. The results obtained from DIgSILENT/PowerFactory are compared with those obtained using the sequence network analysis developed in this paper. The magnitude of negative- sequence voltages obtained for Fig. 4(a) and (b) is presented in Fig. 4(c) and (d) respectively. It can be seen that the results obtained by the two methodologies overlap, which validates the accuracy of the results obtained by the proposed sequence network based method. Comparing between Fig. 4 (c) and (d) it can be seen that $\left|\boldsymbol{U}^{-}\right|$increases slightly when extra branches are added to the original network. However, the consequent variation of negativesequence voltages caused by the network topology change is small, as discussed in Section 2.1.
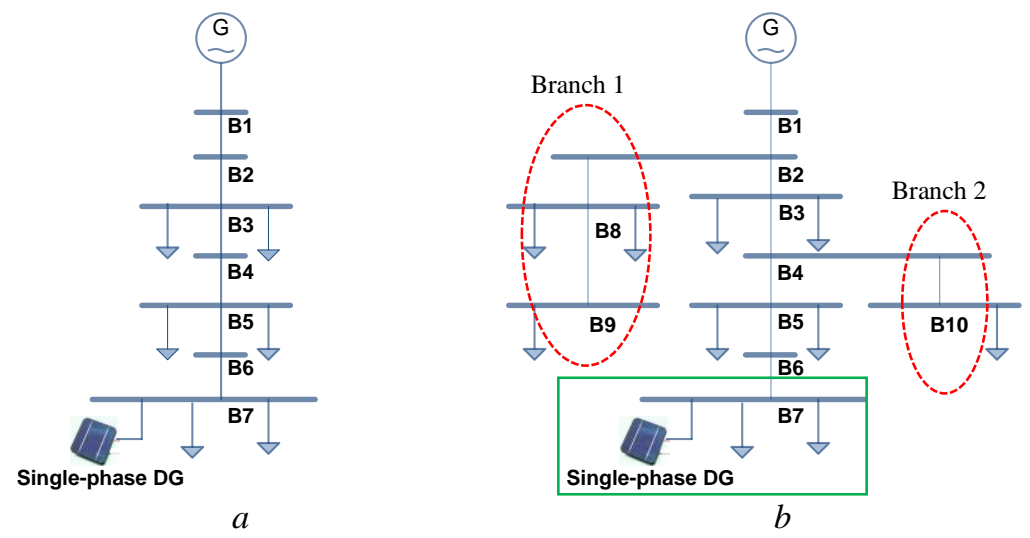


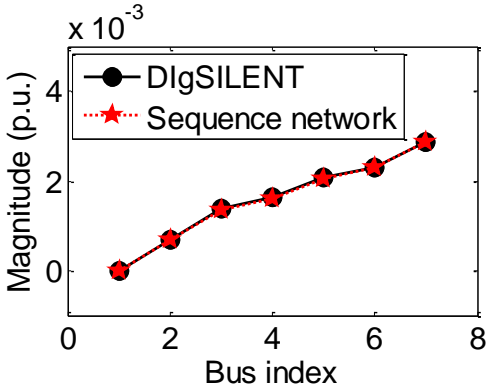

$c$

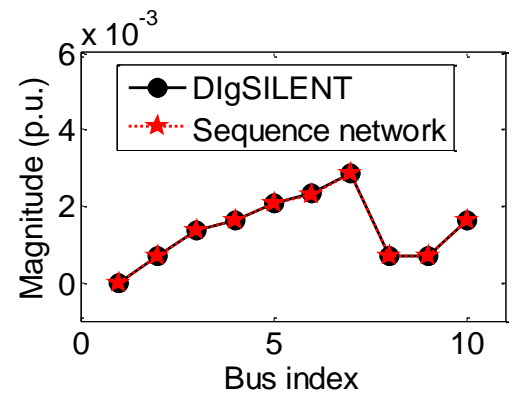

$d$

Fig. 4. Single-line diagrams of considered test feeders and the obtained magnitude of sequence voltages a With one feeder b With extra branches c Negative-sequence voltage for Fig. 4(a)

d Negative-sequence voltage for Fig. 4(b)

\subsection{Correlation between symmetrical components and open circuit voltage $U$}

3.1.1 Variation of line impedance: As discussed in Section 2.1, if line impedance is modified, both Thévenin equivalent impedance of the sequence networks and the open circuit voltage, i.e. $U$ in (15), vary accordingly. The open circuit voltage and the Thévenin equivalent impedance of the negative-sequence network are presented as a function of the line impedance, as shown in Fig. 5(a) and (b) respectively. It can be seen that as line impedance increases, the open circuit voltage decreases and the Thévenin equivalent impedance increases. Although both of them impact the magnitude of sequence voltages, as seen from (15), their influence capability varies. The variation of the open circuit voltage and equivalent impedance, as a response to the variation of line impedance, can be presented by their difference (i.e., distance in y-axis) from the leftmost points in Fig. 5(a) and (b), as presented in Fig. 6(a). Since actual units, i.e. Volt and Ohm are used in (15), actual units are used in Fig. 6(a). It can be seen that if the line impedance is modified, the consequent variation of the open circuit voltage is much larger than that of the equivalent impedance. In other words, in (15), open circuit voltage $U$ is the main factor that determines the variation trend of the magnitude of negative-sequence voltage. To observe the influence of the variation of line impedance on the variation of symmetrical components, the magnitude of both positive- and negative-sequence voltages is presented as a function of the line impedance, as shown in Fig. 5(c) and (d). It confirms the conclusion in Section 2.1, i.e., as the line impedance increases, the magnitude of negativesequence voltage increases and the magnitude of positive-sequence voltage decreases. It can be seen from Fig. 5 that as line impedance increases, $U$ decreases, consequently $\left|\boldsymbol{U}^{+}\right|$decreases and $\left|\boldsymbol{U}^{-}\right|$increases, which is in agreement with the discussion given in Section 2.1. 


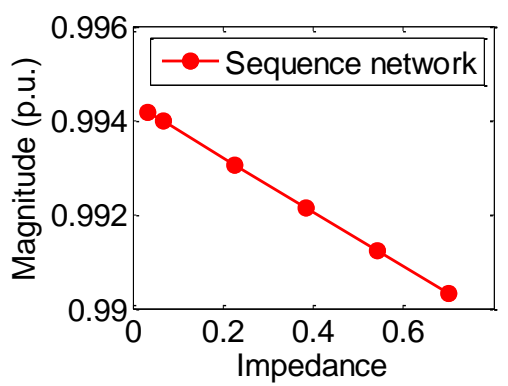

$a$

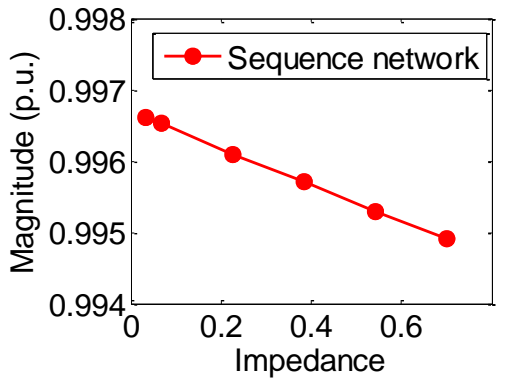

$c$

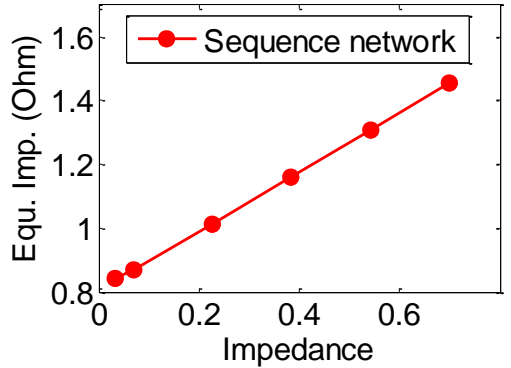

$b$

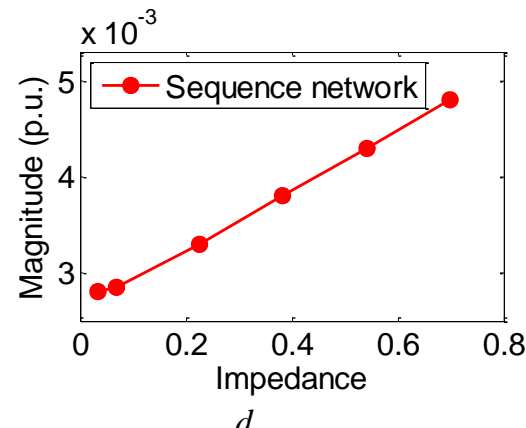

Fig. 5. U, Thévenin equivalent impedance and sequence voltages against line impedance a Open circuit voltage magnitude b Thévenin equivalent impedance c Positive-sequence voltage at B7 d Negative-sequence voltage at B7

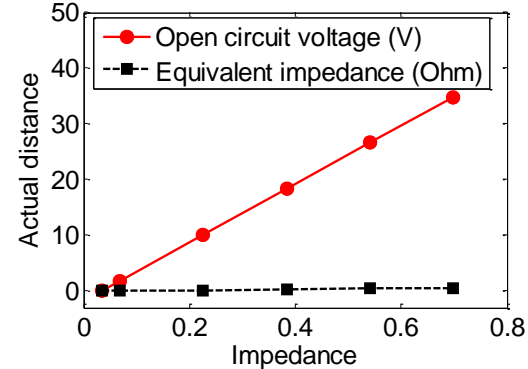

$a$

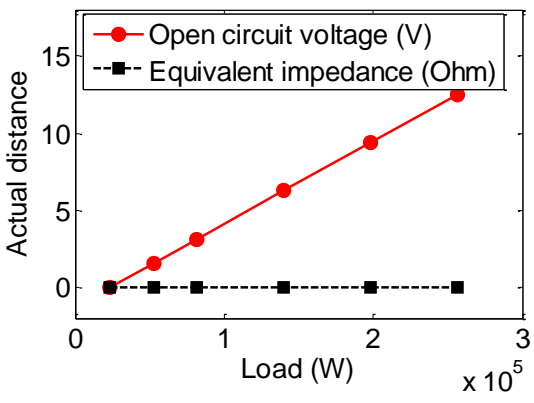

b

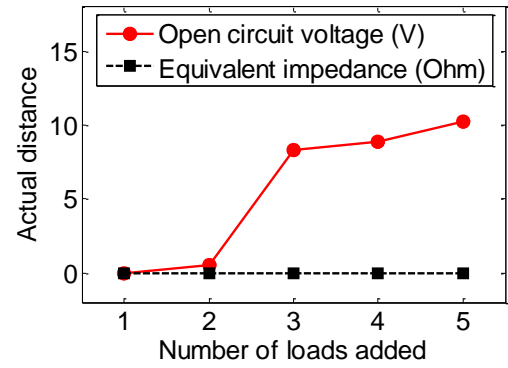

$c$

Fig. 6. The variation of $U$ and equivalent impedance

a Against line impedance

b Against load demand

c Against the number of loads added 
3.1.2 Variation of load demand: As discussed in Section 2.1, the total equivalent impedance and open circuit voltage $U$ in (15) vary as load demand varies. The open circuit voltage and the equivalent impedance of the negative-sequence network are presented as a function of the load demand, as shown in Fig. 7(a) and (b) respectively. It can be seen that as load demand increases, open circuit voltage decreases, and Thévenin equivalent impedance decreases, due to the decrease in $\boldsymbol{Z}_{\text {Load } i}$. Both open-circuit voltage and equivalent impedance impact the magnitude of sequence voltages in (15). The variation of the actual values of the two variables is presented in Fig. 6(b). It can be seen that when load demand is modified, the consequent variation of open circuit voltage is much larger than that of the equivalent impedance. Based on (15), it can be seen that open circuit voltage $U$ is the main factor that determines the variation trend of the magnitude of sequence voltages. Comparing between Fig. 6(a) and (b), it can be seen that the load variation has smaller influence on open circuit voltage and equivalent impedance compared with the variation of line impedance, which is in agreement with the analysis given in Section 2.1. The magnitude of both sequence voltages is represented as a function of the load demand, as shown in Fig. 7(c) and (d), which confirms the discussion in Section 2.1 that the magnitude of negative-sequence voltage increases and the magnitude of positive-sequence voltage decreases as the load demand increases. Comparing between Fig. 5 (c-d) and 7(c-d), it can be seen that line impedance has greater influence on the negativesequence voltage than the load variation, due to the fact that open circuit voltage and the Thévenin equivalent impedance are mainly determined by line impedance rather than load impedance.
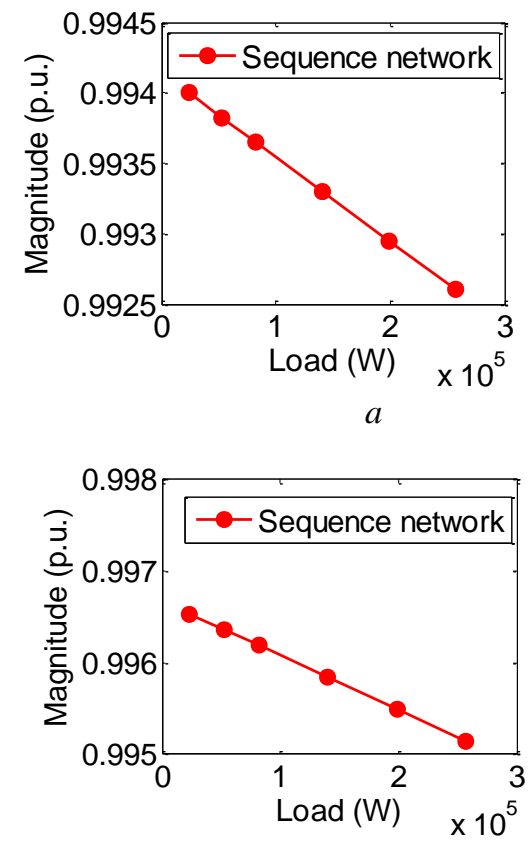

C
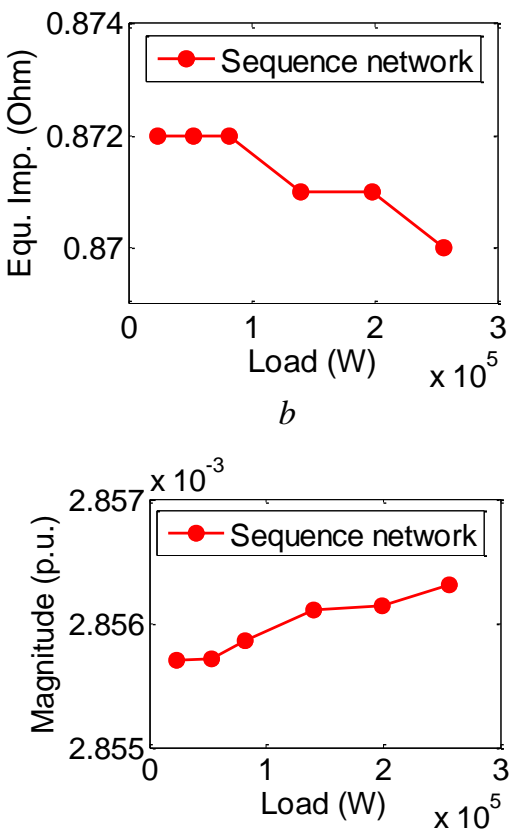

$d$

Fig. 7. U, Thévenin equivalent impedance and sequence voltage against the load demand a Open circuit voltage magnitude 
b Thévenin equivalent impedance

c Positive-sequence voltage at bus B7

d Negative-sequence voltage at bus B7

3.1.3 Variation of network topology: The four loads locating on the extra branches are added to the basic network one by one following the order: left load at B8, right load at B8, load at B9 and load at bus B10. The open circuit voltage $U$ and the equivalent impedance of the negative-sequence network are also presented as a function of the number of additional loads included in the network, as given in Fig. 8(a) and (b), which shows that the influence of the network topology on the open circuit voltage and equivalent impedance is small, compared with the influence of line impedance variation as given in Fig. 5(a) and (b). The variation of the actual values of the open circuit voltage and equivalent impedance is presented in Fig. 6(c) which shows that open circuit voltage $U$ is the main factor that determines the variation trend of the magnitude of sequence voltages compared with the equivalent impedance. $\left|\boldsymbol{U}^{+}\right|$and $\left|\boldsymbol{U}^{-}\right|$at B7 obtained against the number of the additional loads included in the network are shown in Fig. 8(c) and (d) respectively. It can be seen that $\left|\boldsymbol{U}^{-}\right|$increases and $\left|\boldsymbol{U}^{+}\right|$decreases slightly as more loads are added to the new branches. The variation of sequence voltages due to the inclusion of additional loads is small. It can be seen that the influence of network topology on sequence voltage is similar than that of load demand presented in Section 2.1, i.e. loads do not affect the results much.
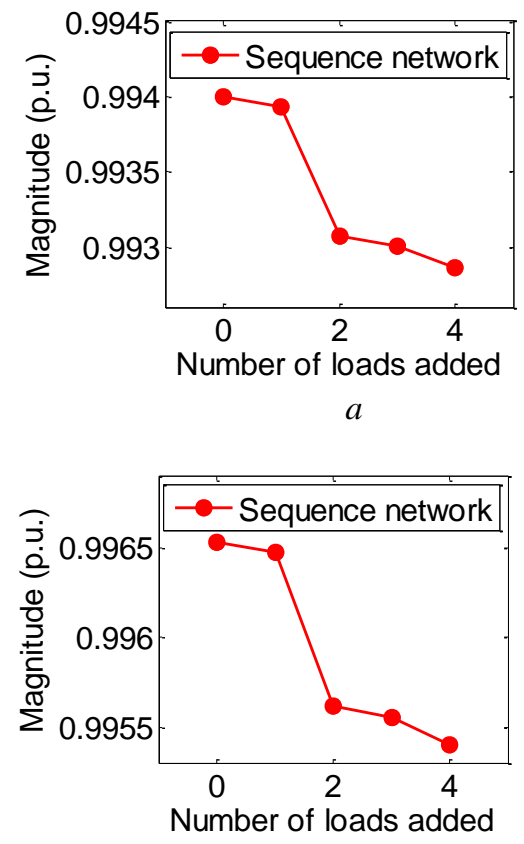

$c$

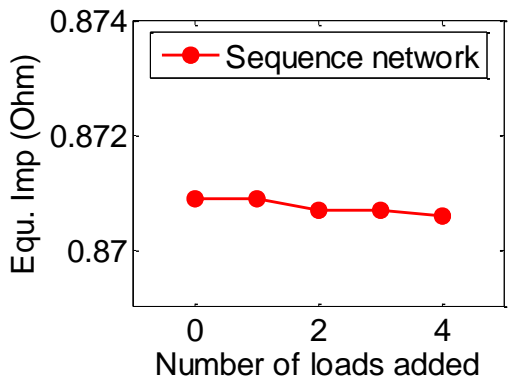

$b$

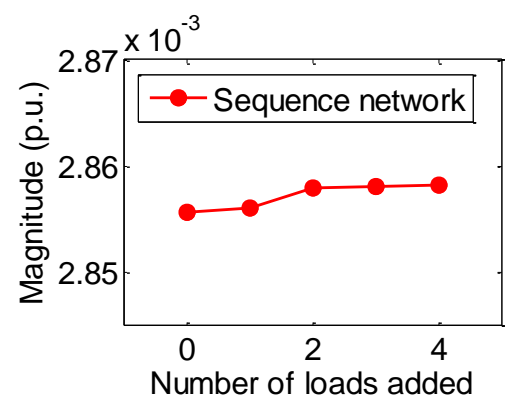

$d$

Fig. 8. U, Thévenin equivalent impedance and sequence voltages against the number of loads added a Open circuit voltage magnitude b Thévenin equivalent impedance c Positive-sequence voltage at bus B7 
d Negative-sequence voltage at bus B7

\subsection{Propagation of negative-sequence voltage}

An unbalance load, whose load demand at phase $\mathrm{A}$ is larger than that at phases $\mathrm{B}$ and $\mathrm{C}$, is connected at B6. The extra load demand at phase A is equal to the power generated by DG. $\left|\boldsymbol{U}^{-}\right|$of all buses is presented in Fig. 9(a). It can be seen that $\left|\boldsymbol{U}^{-}\right|$propagates from B7. When reaching B6 (i.e. the point connected with unbalance load), $\boldsymbol{U}^{-}$is mitigated significantly. As discussed in Section 2.2, the difference between $\boldsymbol{I}_{\mathrm{DA}}^{-1}$ and $\boldsymbol{I}_{\mathrm{FA}}^{-}$determines the unbalance severity at bus B6. In Fig. 9(a), the equivalent current source obtained as the combination of current sources $\boldsymbol{I}_{\mathrm{DA}}^{-\prime}$ and $\boldsymbol{I}_{\mathrm{FA}}^{-}$generates small volume of current. With the small unbalance current source, the unbalance issue is less severe at connecting point $a^{\prime \prime}$. Since $\boldsymbol{I}_{\mathrm{DA}}^{-\prime}$ and $\boldsymbol{I}_{\mathrm{FA}}^{-}$are not exactly the same, $\boldsymbol{U}^{-}$is not completely eliminated at point $a^{\prime \prime}$. As for point $a^{\prime}$, its negative-sequence voltage is mitigated accordingly due to the propagated effect of $\boldsymbol{I}_{\mathrm{FA}}^{-}$. To model the case of $\boldsymbol{I}_{\mathrm{FA}}^{-} \gg \boldsymbol{I}_{\mathrm{DA}}^{-1}$, the extra load demand absorbed at phase $\mathrm{A}$ is increased to twice of the power generated by DG. $\left|\boldsymbol{U}^{-}\right|$of all buses is presented in Fig. 9(b). It can be seen that in this case $\left|\boldsymbol{U}^{-}\right|$at B6 is larger than that of other buses, as $\boldsymbol{I}_{\mathrm{FA}}^{-}$is the dominant unbalance source, compared to $\boldsymbol{I}_{\mathrm{DA}}^{-} \cdot\left|\boldsymbol{U}^{-}\right|$in Fig. 9(b) is larger than that in Fig. 9(a). Comparing the two cases of different settings for unbalance load, it can be seen that proper distribution of load demand along the feeder can mitigate the unbalance propagation resulting from negative-sequence voltage caused by DG. To validate the application of the proposed approach in a more generic scheme with multiple DGs, another two, single-phase connected DGs are added in the network, apart from the DG connected to phase A at B7. The two DGs are connected to phase $\mathrm{B}$ at B6 and to phase C at B4 respectively, injecting the same amount of power as that by the DG connected at B7. $\left|\boldsymbol{U}^{-}\right|$of all buses obtained by both approaches is presented in Fig. 9(c). It can be seen that $\left|\boldsymbol{U}^{-}\right|$in Fig. 9(c) is larger than that in Fig. 9(a). Similar to the settings in case 1, the extra amount of the power drawn from phase A at B6 by the unbalance load is the same as that injected by DG connected to B7. Norton's theorem is applied to the negative-sequence network of the circuit section encircled by green solid line in Fig. 4(b), and its equivalent current source caused by the DG connected at B7 injects current to point $a^{\prime \prime}$ at B6, denoted as $\boldsymbol{I}_{\mathrm{DA}}^{-\prime} \cdot \boldsymbol{I}_{\mathrm{FA}}^{-}$and $\boldsymbol{I}_{\mathrm{DA}}^{-\prime}$ are very similar, derived based on (2) and (16), but flow in opposite direction, as discussed in Section 2.2 and shown in Fig. 3(c). Since they are not exactly the same, they cannot cancel each other completely at B6. Their residual part (i.e., the combined effect between $\boldsymbol{I}_{\mathrm{DA}}^{-\prime}$ and $\left.\boldsymbol{I}_{\mathrm{FA}}^{-}\right)$together with current $\boldsymbol{I}_{\mathrm{DB}}^{-}$caused by the DG connected at B6 results in peak $\left|\boldsymbol{U}^{-}\right|$at B6. 

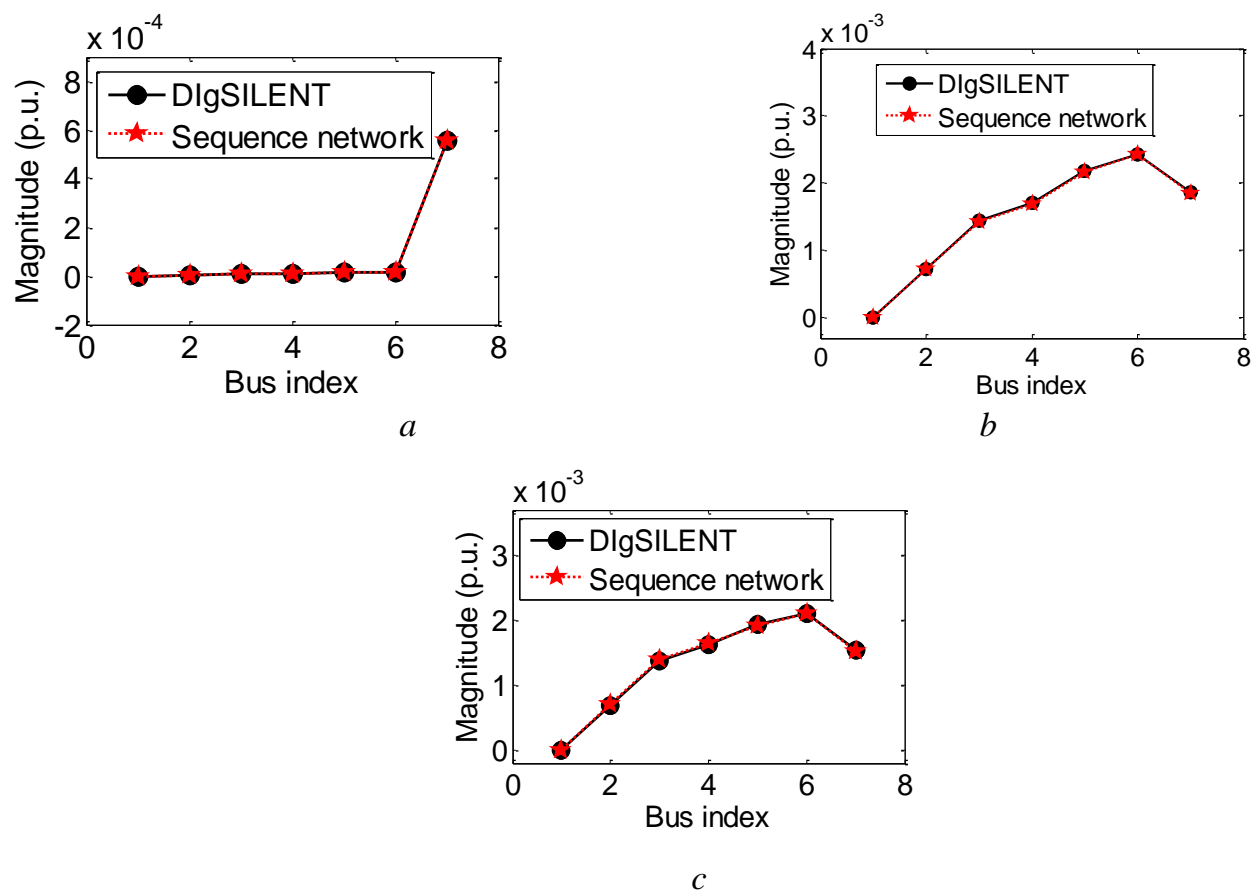

Fig. 9. $\left|\boldsymbol{U}^{-}\right|$when connecting an unbalanced load at B6 and multiple single-phase connected DGs a case 1

$\mathrm{b}$ case 2

c case 3

The proposed approach is further validated on a 96-bus section of a generic UK distribution network $[19,20]$, which is likely to be exposed to unbalance phenomena if unbalance sources exist in the network. The single line diagram of the network is given in Fig. 10 (a), and the relevant network parameters in Appendix A. In the study, nine single-phase connected DGs are distributed around the network, and connected to different phases and different buses in the network. The heat-map is employed to present the unbalance propagation in the network based on the results obtained from the proposed approach, as given in Fig. 10(a). It can be seen that the areas exposed to higher $\left|\boldsymbol{U}^{-}\right|$, i.e., the areas encircled by the red dashed lines, can be easily identified. $\left|\boldsymbol{U}^{-}\right|$propagates from the circled area and gradually diminishes along the feeder towards high voltage level. Additionally, the $\left|\boldsymbol{U}^{-}\right|$of the buses along the main feeder downstream to the DGs, obtained by both approaches is given in Fig. 10(b). The results are in line with the results presented in the heat-map. 

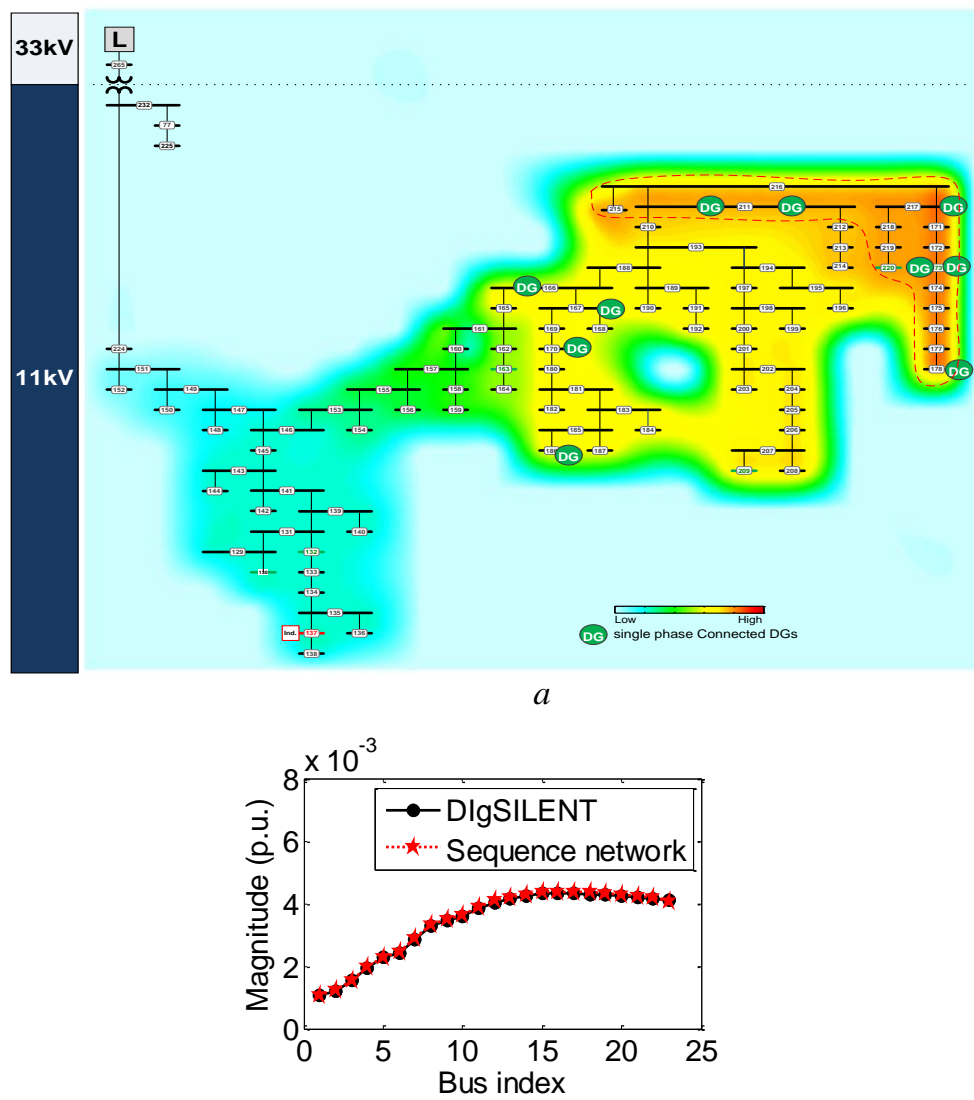

b

Fig. 10. $\left|\boldsymbol{U}^{-}\right|$in $96-$ bus section of a generic UK distribution network a heat-map

b comparison between the two approaches

\section{Conclusion}

The paper presented sequence network based methodology to analyse the unbalance severity and propagation in distribution networks with single-phase DG. The methodology simplifies the analysis of unbalanced conditions in a polyphase system and provides the straightforward visualization of the propagation of sequence voltages using single-line diagrams. The detailed procedure of deriving the sequence circuit formulae, and their use in identifying the critical components that affect the unbalance severity and propagation, are provided in the paper. The methodology and the conclusions derived from the sequence network analysis are validated by comparing the obtained results with those obtained from simulations in DIgSILENT/PowerFactory using phase voltage based methodology. The analysis suggests that the contribution of different network parameters on symmetrical components at the DG connection points varies, and that the variation of the line impedance has more influence on variation of symmetrical components than the variation of load demand and network topology. The trend of variation of the symmetrical voltages as a result of the connection of DG can be estimated by observing the variation of 
measured open circuit voltage at the potential DG connection point. The distribution of line impedances along the feeder impacts the unbalance propagation appreciably, and the impedance of the lines closer to the DG connected point has greater influence. Finally, the distribution of load demand along the feeder can mitigate the unbalance propagation resulting from the negative-sequence voltage caused by DG.

\section{Acknowledgments}

This work was supported by SuSTAINABLE Project under Grant 308755.

\section{References}

[1] Von, J.A., Banerjee, B.:'Assessment of voltage unbalance', IEEE Trans. Power Del., 2001, 16, (4), pp. 782 790

[2] Habijan, D., Cavlovic, M., Jaksic, D.:'The issue of asymmetry in low voltage network with distributed geneartion'. Proc. Int. Conf. on Elec. Dist., Stockholm, 2013

[3] Meng, L., Tang, F., Savaghebi, M., et. al.:'Tertiary control of voltage unbalance compensation for optimal power quality in islanded microgrids', IEEE Trans. Energy Conv., 2014, 29, (4), pp. 802-815.

[4] Savaghebi, M., Jalilian, A., Vasquez, J.C., et. al.:"Secondary control scheme for voltage unbalance compensation in an islanded droop-controlled microgrid', IEEE Trans. Smart Grid, 2012, 3, (2), pp. 797-807

[5] IEC 61000-4-30:2003, 'Testing and measurement techniques - Power quality measurement methods' (2003)

[6] EN 50160:2004,'Voltage disturbances standard EN 50160 - voltage characteristics in public distribution systems' (2004)

[7] Neto, A.F.T., Cunha, G.P.L., Mendonca, M.V.B., et. al.:'A comparative evaluation of methods for analysis of propagation of unbalance in electric systems’. Proc. IEEE PES Trans. and Dist., Orlando, 2012, pp. 1-8

[8] Yan, R., Saha, T.K.:'Investigation of voltage imbalance due to distribution network unbalanced line configurations and load levels', IEEE Trans. Power Syst., 2013, 28, (2), pp. 1829-1838

[9] Abdel-Akher, M., Nor, K.M., Rashid, A.H.A.:'Improved three-phase power-flow methods using sequence components’, IEEE Trans. Power Syst., 2005, 20, (3), pp. 1389-1397

[10] Howard, D.F., Habetler, T.G., Harley, R.G.:'Improved sequence network model of wind turbine generators for short-circuit studies', IEEE Trans. Ener. Conv., 2012, 27, (4), pp. 968-977

[11] Bergen, A. R., and Vittal, V.:'Power Systems Analysis’'(Prentice-Hall, Inc, Second Edition, 2000)

[12] Liu, Z.X., Milanović, J.V.:'Probabilistic estimation of propagation of unbalance in distribution network with asymmetrical loads'. Proc. 8th Medi. Conf. on Power Gen., Tran., Distri. and Ene. Conver., Cagliari, 2012, pp. $1-6$

[13] Chindris, M., Cziker, A., Miron, A., et al.:'Propagation of unbalance in electric power systems’. Proc. 9th Int. Conf. on Electr. Power Quality and Utilisation, 2007, pp. 1-5 
[14] Liu, Z., Milanović, J.V.:'Probabilistic estimation of voltage unbalance in MV distribution networks with unbalanced load', IEEE Trans. Power Del., 2015, 30, (2), pp. 693-703

[15] Bollen, M.H., Hassan, F.:'Integration of Distributed Generation in the Power System' (John Wiley \& Sons, 2011)

[16] Dugan, R.C., McGranaghan, M.F., Santoso, S., et al.:'Electrical Power Systems Quality' (McGraw Hill, Second Edition, 2003)

[17] Kersting W.H.:'Distribution System Modeling and Analysis’'(CRC Press, 3 edition, 2012)

[18] Mora, J.M.A.:'Monitor placement for estimation of voltage sags in power systems' Ph.D. thesis, University of Manchester, 2012

[19] Liao, H.L., Liu, Z., Milanović, J.V., et al.:'Optimisation framework for development of cost-effective monitoring in distribution networks', IET Gen., Trans. \& Dis., 2016, 10, (1), pp. 240-246

[20] Liao, H.L., Abdelrahman, S., Guo, Y., and Milanović, J.V.:'Identification of weak areas of power network based on exposure to voltage sags-Part II: assessment of network performance using sag severity index', IEEE Trans. Power Del. 2015, 30, (6), pp. 2401-2409

\section{Appendix}

Table 1 Input data (buses and lines) for 96-bus test network

\begin{tabular}{|c|c|c|c|c|c|}
\hline bus- $i$ & Pd & Qd & BaseKV & Vmax & Vmin \\
\hline 1 & 0 & 0 & 33 & 1.06 & 0.94 \\
\hline 2 & 0.9612 & 0.1756 & 11 & 1.06 & 0.94 \\
\hline 3 & 0 & 0 & 11 & 1.06 & 0.94 \\
\hline 4 & 0 & 0 & 11 & 1.06 & 0.94 \\
\hline 5 & 0.6706 & 0.1313 & 11 & 1.06 & 0.94 \\
\hline 6 & 0 & 0 & 11 & 1.06 & 0.94 \\
\hline 7 & 0 & 0 & 11 & 1.06 & 0.94 \\
\hline 8 & 0 & 0 & 11 & 1.06 & 0.94 \\
\hline 9 & 0.0789 & 0.0197 & 11 & 1.06 & 0.94 \\
\hline 10 & 0 & 0 & 11 & 1.06 & 0.94 \\
\hline 11 & 0.0619 & 0.0094 & 11 & 1.06 & 0.94 \\
\hline 12 & 0 & 0 & 11 & 1.06 & 0.94 \\
\hline 13 & 0 & 0 & 11 & 1.06 & 0.94 \\
\hline 14 & 0.1116 & 0.0225 & 11 & 1.06 & 0.94 \\
\hline 15 & 0 & 0 & 11 & 1.06 & 0.94 \\
\hline 16 & 0.0347 & 0.0047 & 11 & 1.06 & 0.94 \\
\hline 17 & 0 & 0 & 11 & 1.06 & 0.94 \\
\hline 18 & 0.0356 & 0.0066 & 11 & 1.06 & 0.94 \\
\hline 19 & 0 & 0 & 11 & 1.06 & 0.94 \\
\hline 20 & 0 & 0 & 11 & 1.06 & 0.94 \\
\hline 21 & 0 & 0 & 11 & 1.06 & 0.94 \\
\hline 22 & 0.032 & 0.0047 & 11 & 1.06 & 0.94 \\
\hline 23 & 0 & 0 & 11 & 1.06 & 0.94 \\
\hline & & & & & \\
\hline
\end{tabular}

\begin{tabular}{|c|c|c|c|c|c|}
\hline from & to & $\mathrm{r}$ & $\mathrm{x}$ & $\mathrm{r}^{0}$ & $\mathrm{x}^{0}$ \\
\hline 2 & 3 & 0.04879 & 0.05058 & 0.29274 & 0.15174 \\
\hline 3 & 4 & 0.09755 & 0.33284 & 0.58530 & 0.99852 \\
\hline 4 & 5 & 0.17322 & 0.07589 & 1.03932 & 0.22767 \\
\hline 4 & 6 & 0.21 & 0.203 & 1.26 & 0.609 \\
\hline 6 & 7 & 0.2451 & 0.10624 & 1.4706 & 0.31872 \\
\hline 6 & 8 & 0.2586 & 0.17673 & 1.5516 & 0.53019 \\
\hline 8 & 9 & 0.34645 & 0.15178 & 2.0787 & 0.45534 \\
\hline 8 & 10 & 0.1293 & 0.08836 & 0.7758 & 0.26508 \\
\hline 10 & 94 & 0.295 & 0.15 & 1.77 & 0.45 \\
\hline 10 & 11 & 0.30169 & 0.20618 & 1.8014 & 0.61854 \\
\hline 11 & 12 & 0.19395 & 0.13255 & 1.1637 & 0.39765 \\
\hline 12 & 13 & 0.17322 & 0.07589 & 1.03932 & 0.22767 \\
\hline 12 & 14 & 0.23705 & 0.162 & 1.4223 & 0.486 \\
\hline 14 & 15 & 0.20787 & 0.09107 & 1.24722 & 0.27321 \\
\hline 14 & 16 & 0.25860 & 0.17673 & 1.55160 & 0.53019 \\
\hline 16 & 17 & 0.13858 & 0.06071 & 0.83148 & 0.18213 \\
\hline 16 & 18 & 0.1293 & 0.08836 & 0.7758 & 0.26508 \\
\hline 18 & 20 & 0.2155 & 0.14727 & 1.293 & 0.44181 \\
\hline 18 & 19 & 0.1724 & 0.11782 & 1.0344 & 0.35346 \\
\hline 19 & 96 & 0.10775 & 0.07364 & 0.6465 & 0.22092 \\
\hline 2 & 27 & 0.05489 & 0.0569 & 0.32934 & 0.1707 \\
\hline 27 & 28 & 0.03881 & 0.104 & 0.23286 & 0.312 \\
\hline 29 & 30 & 0.1293 & 0.08836 & 0.7758 & 0.26508 \\
\hline & & & & & \\
\hline
\end{tabular}




\begin{tabular}{|c|c|c|c|c|c|}
\hline 24 & 0.018 & 0.0028 & 11 & 1.06 & 0.94 \\
\hline 25 & 0 & 0 & 11 & 1.06 & 0.94 \\
\hline 26 & 0.0151 & 0.0028 & 11 & 1.06 & 0.94 \\
\hline 27 & 0.0085 & 0.0009 & 11 & 1.06 & 0.94 \\
\hline 28 & 0.0085 & 0.0009 & 11 & 1.06 & 0.94 \\
\hline 29 & 0 & 0 & 11 & 1.06 & 0.94 \\
\hline 30 & 0.016 & 0.0019 & 11 & 1.06 & 0.94 \\
\hline 31 & 0.017 & 0.0028 & 11 & 1.06 & 0.94 \\
\hline 32 & 0.0226 & 0.0047 & 11 & 1.06 & 0.94 \\
\hline 33 & 0 & 0 & 11 & 1.06 & 0.94 \\
\hline 34 & 0.0198 & 0.0028 & 11 & 1.06 & 0.94 \\
\hline 35 & 0.0075 & 0.0009 & 11 & 1.06 & 0.94 \\
\hline 36 & 0 & 0 & 11 & 1.06 & 0.94 \\
\hline 37 & 0 & 0 & 11 & 1.06 & 0.94 \\
\hline 38 & 0 & 0 & 11 & 1.06 & 0.94 \\
\hline 39 & 0.0075 & 0.0009 & 11 & 1.06 & 0.94 \\
\hline 40 & 0.0442 & 0.0094 & 11 & 1.06 & 0.94 \\
\hline 41 & 0 & 0 & 11 & 1.06 & 0.94 \\
\hline 42 & 0 & 0 & 11 & 1.06 & 0.94 \\
\hline 43 & 0.0047 & 0.0065 & 11 & 1.06 & 0.94 \\
\hline 44 & 0.0047 & 0.0007 & 11 & 1.06 & 0.94 \\
\hline 45 & 0.0047 & 0.0007 & 11 & 1.06 & 0.94 \\
\hline 46 & 0 & 0 & 11 & 1.06 & 0.94 \\
\hline 47 & 0 & 0 & 11 & 1.06 & 0.94 \\
\hline 48 & 0.039 & 0.0076 & 11 & 1.06 & 0.94 \\
\hline 49 & 0.02 & 0.0029 & 11 & 1.06 & 0.94 \\
\hline 50 & 0 & 0 & 11 & 1.06 & 0.94 \\
\hline 51 & 0 & 0 & 11 & 1.06 & 0.94 \\
\hline 52 & 0 & 0 & 11 & 1.06 & 0.94 \\
\hline 53 & 0.0085 & 0.0009 & 11 & 1.06 & 0.94 \\
\hline 54 & 0 & 0 & 11 & 1.06 & 0.94 \\
\hline 55 & 0.0114 & 0.0209 & 11 & 1.06 & 0.94 \\
\hline 56 & 0 & 0 & 11 & 1.06 & 0.94 \\
\hline 57 & 0.019 & 0.0028 & 11 & 1.06 & 0.94 \\
\hline 58 & 0.0398 & 0.0085 & 11 & 1.06 & 0.94 \\
\hline 59 & 0.0076 & 0.001 & 11 & 1.06 & 0.94 \\
\hline 60 & 0 & 0 & 11 & 1.06 & 0.94 \\
\hline 61 & 0.0345 & 0.0048 & 11 & 1.06 & 0.94 \\
\hline 62 & 0.1312 & 0.0316 & 11 & 1.06 & 0.94 \\
\hline 63 & 0.0048 & 0.0007 & 11 & 1.06 & 0.94 \\
\hline 64 & 0.0183 & 0.0029 & 11 & 1.06 & 0.94 \\
\hline 65 & 0 & 0 & 11 & 1.06 & 0.94 \\
\hline 66 & 0.0348 & 0.0058 & 11 & 1.06 & 0.94 \\
\hline 67 & 0.0203 & 0.0029 & 11 & 1.06 & 0.94 \\
\hline 68 & 0 & 0 & 11 & 1.06 & 0.94 \\
\hline 69 & 0.0127 & 0.0019 & 11 & 1.06 & 0.94 \\
\hline
\end{tabular}

\begin{tabular}{|c|c|c|c|c|c|}
\hline 30 & 31 & 0.19395 & 0.13255 & 1.1637 & 0.39765 \\
\hline 31 & 35 & 0.2586 & 0.17673 & 1.5516 & 0.53019 \\
\hline 35 & 36 & 0.06465 & 0.04418 & 0.3879 & 0.13254 \\
\hline 36 & 37 & 0.27244 & 0.04012 & 1.63464 & 0.12036 \\
\hline 37 & 38 & 0.16346 & 0.02407 & 0.98076 & 0.07221 \\
\hline 38 & 39 & 0.0862 & 0.05891 & 0.5172 & 0.17673 \\
\hline 39 & 40 & 0.15085 & 0.10309 & 0.9051 & 0.30927 \\
\hline 40 & 41 & 0.27244 & 0.04012 & 1.63464 & 0.12036 \\
\hline 31 & 32 & 0.27716 & 0.12142 & 1.66296 & 0.36429 \\
\hline 32 & 33 & 0.48502 & 0.21249 & 2.91012 & 1.27494 \\
\hline 33 & 34 & 0.22621 & 0.04686 & 1.35726 & 0.14058 \\
\hline 94 & 95 & 0.20787 & 0.09107 & 1.24722 & 0.27321 \\
\hline 94 & 92 & 0.354 & 0.18 & 2.124 & 0.54 \\
\hline 92 & 93 & 0.24251 & 0.10624 & 1.45506 & 0.31872 \\
\hline 92 & 89 & 0.27716 & 0.12142 & 1.66296 & 0.36426 \\
\hline 89 & 90 & 0.25860 & 0.17673 & 1.5516 & 0.53019 \\
\hline 90 & 91 & 0.31180 & 0.1366 & 1.8708 & 0.4098 \\
\hline 89 & 88 & 0.11149 & 0.07376 & 0.66894 & 0.22128 \\
\hline 88 & 84 & 0.34612 & 0.20653 & 2.07672 & 0.61959 \\
\hline 84 & 85 & 0.15608 & 0.10326 & 0.93648 & 0.30978 \\
\hline 85 & 86 & 0.22298 & 0.14752 & 1.33788 & 0.44256 \\
\hline 86 & 87 & 0.21350 & 0.09126 & 1.281 & 0.27378 \\
\hline 84 & 83 & 0.34479 & 0.23564 & 2.06874 & 0.70692 \\
\hline 83 & 70 & 0.1293 & 0.08836 & 0.7758 & 0.26508 \\
\hline 70 & 71 & 0.118 & 0.06 & 0.708 & 0.18 \\
\hline 71 & 72 & 0.20787 & 0.09107 & 1.24722 & 0.2732 \\
\hline 71 & 73 & 0.236 & 0.12 & 1.416 & 0.36 \\
\hline 73 & 74 & 0.177 & 0.09 & 1.062 & 0.27 \\
\hline 74 & 75 & 0.09401 & 0.03595 & 0.56406 & 0.10785 \\
\hline 75 & 76 & 0.177 & 0.09 & 1.062 & 0.27 \\
\hline 76 & 77 & 0.236 & 0.12 & 1.416 & 0.36 \\
\hline 76 & 78 & 0.354 & 0.18 & 2.124 & 0.54 \\
\hline 78 & 79 & 0.354 & 0.18 & 2.124 & 0.54 \\
\hline 78 & 80 & 0.27716 & 0.12142 & 1.66296 & 0.36426 \\
\hline 80 & 81 & 0.2135 & 0.09126 & 1.281 & 0.27378 \\
\hline 80 & 82 & 0.53374 & 0.22816 & 3.20244 & 0.68448 \\
\hline 20 & 21 & 0.19395 & 0.13255 & 1.1637 & 0.39765 \\
\hline 21 & 22 & 0.30169 & 0.20618 & 1.81014 & 0.61854 \\
\hline 22 & 23 & 0.09 & 0.087 & 0.54 & 0.261 \\
\hline 23 & 26 & 0.13161 & 0.05033 & 0.78966 & 0.15099 \\
\hline 23 & 24 & 0.36 & 0.348 & 2.16 & 1.044 \\
\hline 24 & 25 & 0.12 & 0.116 & 0.72 & 0.348 \\
\hline 70 & 65 & 0.1724 & 0.11782 & 0.0344 & 0.35346 \\
\hline 65 & 66 & 0.20787 & 0.09107 & 1.24722 & 0.27321 \\
\hline 66 & 67 & 0.27716 & 0.12142 & 1.66296 & 0.36426 \\
\hline 66 & 68 & 0.41574 & 0.18213 & 2.4944 & 0.54639 \\
\hline
\end{tabular}




\begin{tabular}{|c|c|c|c|c|c|}
\hline 70 & 0 & 0 & 11 & 1.06 & 0.94 \\
\hline 71 & 0.0206 & 0.0039 & 11 & 1.06 & 0.94 \\
\hline 72 & 0 & 0 & 11 & 1.06 & 0.94 \\
\hline 73 & 0.0029 & 0.0004 & 11 & 1.06 & 0.94 \\
\hline 74 & 0 & 0 & 11 & 1.06 & 0.94 \\
\hline 75 & 0.0029 & 0.0004 & 11 & 1.06 & 0.94 \\
\hline 76 & 0 & 0 & 11 & 1.06 & 0.94 \\
\hline 77 & 0.0213 & 0.0068 & 11 & 1.06 & 0.94 \\
\hline 78 & 0 & 0 & 11 & 1.06 & 0.94 \\
\hline 79 & 0.0096 & 0.0019 & 11 & 1.06 & 0.94 \\
\hline 80 & 0 & 0 & 11 & 1.06 & 0.94 \\
\hline 81 & 0 & 0 & 11 & 1.06 & 0.94 \\
\hline 82 & 0.0353 & 0.0048 & 11 & 1.06 & 0.94 \\
\hline 83 & 0.018 & 0.0028 & 11 & 1.06 & 0.94 \\
\hline 84 & 0.017 & 0.0028 & 11 & 1.06 & 0.94 \\
\hline 85 & 0 & 0 & 11 & 1.06 & 0.94 \\
\hline 86 & 0.0094 & 0.0009 & 11 & 1.06 & 0.94 \\
\hline 87 & 0.8682 & 0.2853 & 11 & 1.06 & 0.94 \\
\hline 88 & 0 & 0 & 11 & 1.06 & 0.94 \\
\hline 89 & 0.0431 & 0.0077 & 11 & 1.06 & 0.94 \\
\hline 90 & 0 & 0 & 11 & 1.06 & 0.94 \\
\hline 91 & 0 & 0 & 11 & 1.06 & 0.94 \\
\hline 92 & 0.0091 & 0.001 & 11 & 1.06 & 0.94 \\
\hline 93 & 0 & 0 & 11 & 1.06 & 0.94 \\
\hline 94 & 0.0201 & 0.003 & 11 & 1.06 & 0.94 \\
\hline 95 & 0 & 0 & 11 & 1.06 & 0.94 \\
\hline 96 & 0.0189 & 0.003 & 11 & 1.06 & 0.94 \\
\hline
\end{tabular}

\begin{tabular}{|c|c|c|c|c|c|}
\hline 68 & 69 & 0.27716 & 0.12142 & 1.66296 & 0.36426 \\
\hline 65 & 48 & 0.30169 & 0.20618 & 1.81014 & 0.61854 \\
\hline 48 & 47 & 0.2586 & 0.17673 & 1.5516 & 0.53019 \\
\hline 47 & 43 & 0.1293 & 0.08836 & 0.7758 & 0.26508 \\
\hline 43 & 30 & 0.2155 & 0.14727 & 1.293 & 0.44181 \\
\hline 43 & 44 & 0.18258 & 0.07622 & 1.09548 & 0.22866 \\
\hline 44 & 45 & 0.29213 & 0.12195 & 1.75278 & 0.36585 \\
\hline 45 & 46 & 0.4382 & 0.17673 & 2.6292 & 0.53019 \\
\hline 48 & 49 & 0.36517 & 0.15244 & 2.19102 & 0.45732 \\
\hline 49 & 50 & 0.14607 & 0.06098 & 0.87642 & 0.18294 \\
\hline 49 & 51 & 0.17322 & 0.07589 & 1.03932 & 0.22767 \\
\hline 51 & 52 & 0.3118 & 0.1366 & 1.8708 & 0.4098 \\
\hline 50 & 53 & 0.25562 & 0.10671 & 1.53372 & 0.32013 \\
\hline 53 & 54 & 0.20787 & 0.09107 & 1.24722 & 0.27321 \\
\hline 53 & 55 & 0.18258 & 0.07622 & 1.09548 & 0.22866 \\
\hline 57 & 58 & 0.09401 & 0.03595 & 0.56406 & 0.10785 \\
\hline 57 & 59 & 0.4382 & 0.18293 & 2.6292 & 0.5487 \\
\hline 59 & 60 & 0.2191 & 0.09146 & 1.3146 & 0.27447 \\
\hline 60 & 61 & 0.07521 & 0.02876 & 0.4512 & 0.08628 \\
\hline 61 & 62 & 0.14607 & 0.06098 & 0.8764 & 0.18294 \\
\hline 62 & 63 & 0.29213 & 0.12195 & 1.75278 & 0.36585 \\
\hline 62 & 64 & 0.40168 & 0.16768 & 2.41008 & 0.50304 \\
\hline 55 & 56 & 0.29213 & 0.12195 & 1.75278 & 0.36585 \\
\hline 56 & 57 & 0.25562 & 0.1067 & 1.53372 & 0.32013 \\
\hline 41 & 42 & 0.49039 & 0.07222 & 2.94234 & 0.21666 \\
\hline & & & & & \\
\hline 50 &
\end{tabular}

